(c) 2016 IEEE. Personal use of this material is permitted. Permission from IEEE must be obtained for all other users, including reprinting/ republishing this material for advertising or promotional purposes, creating new collective works for resale or redistribution to servers or lists, or reuse of any copyrighted components of this work in other works. 


\title{
Model-Based Control for a Three-Phase Shunt Active Power Filter
}

\author{
Ramon Guzman, Luis Garcia de Vicuña, Javier Morales, Miguel Castilla and Jaume Miret
}

\begin{abstract}
This paper presents a robust model-based control in natural frame for a three-phase shunt active power filter. For the proposed control method a linear converter model is deduced. Then, this model is used in a Kalman filter in order to estimate the system state-space variables. Even though the states estimation do not match the variables of the real system, it has allowed to design three sliding mode controllers providing the following features to the closed loop system: a) robustness due to the fact that control specifications are met independently of any variation in the system parameters; b) noise immunity, since a Kalman filter is applied; c) a lower THD of the current delivered by the grid compared with the standard solution using measured variables; $d$ ) the fundamental component of the voltage at point of common coupling is estimated even in the case of a distorted grid; and e) a reduction in the number of sensors. Thanks to this solution the sliding surfaces for each controller are independent. This decoupling property of the three controllers allows using a fixed switching frequency algorithm that ensures a perfect current control. Finally, experimental results validate the proposed control strategy and illustrate all its interesting features.
\end{abstract}

Index Terms-current control, LCL filter, sliding mode control, Kalman filter, voltage sensorless control.

\section{INTRODUCTION}

$\mathbf{T}$ HE use of nonlinear loads is a cause of current harmonics generation which degrade the quality of the power delivered by the grid. This fact causes non desirable effects on the power supply system. Ordinarily, passive power filters are used to suppress current harmonics due to their low cost [1], [2]. However, this kind of filters is sensitive to parameters variation and it may even produce a resonance problem. This resonance may lead to an over-current or over-voltage in the inductor and capacitor filter damaging the passive power filter.

As an alternative, shunt active power filters (SAPFs) have been used to avoid the aforementioned problems [3], [4]. In contrast to passive power filters, SAPFs are a flexible solution to compensate current harmonics generated by different types

Manuscript received August 10, 2015, revised November 16, 2015; accepted February 04, 2016. Date of current version xxxxx x, 2016. Recommended for publication by Associate Editor xxxxx.

Copyright (c) 2016 IEEE. Personal use of this material is permitted. However, permission to use this material for any other purposes must be obtained from the IEEE by sending a request to pubs-permissions@ieee.org.

This work was supported by the Era-net project ELAC2014/ESE0034 and by the Ministry of Economy and Competitiveness of Spain under project PCIN-2015-001.

Ramon Guzman is with the Department of Automatic control, Technical University of Catalonia, 08800 Vilanova i la Geltru, Spain (email:ramon.guzman@upc.edu). Other authors are with the Department of Electronic Engineering, Technical University of Catalonia, 08800 Vilanova i la Geltru, Spain(e-mail:vicuna@eel.upc.edu;javier.morales.lopez@upc.edu; miquel.castilla@upc.edu; jmiret@eel.upc.edu).

Digital Object Identifier xx.xxxx/yyyy.xxxx.xxxxxxx. of nonlinear loads, providing as well as a fast response to load variations.

In addition, the hybrid topology of SAPFs has been suggested in order to improve the filtering process. These kind of active filters incorporate an LC tank with the SAPF in order to reduce the effort of the control algorithm [5]-[7].

It is well known that the main purpose of a SAPF is to generate the harmonics caused by nonlinear loads, with the objective of providing a grid current with a low-harmonic content. Bearing this objective in mind, SAPF control has been widely studied, while considering two different control loops [3]-[5]:an outer voltage loop is designed in order to keep the voltage constant at the output capacitor filter, while the inner current loop is used to track the reference current, which can be generated by means of two different methods. The first method, the direct one, is based on extracting the harmonic content of the load current. Then, the filter current is used in the inner current loop in order to track the load current harmonics [8], [9]. The second method, the indirect one, is based on generating a sinusoidal reference current from the grid-voltage measurement. Then, the grid current is forced to track this sinusoidal current reference and the load current harmonics are provided by the filter inductor current in an indirect way [10]. The main advantages of this second approach are that only one low-bandwidth current sensor is required and a faster transient response is obtained. However, since the reference current is generated from measured voltages at the point of common coupling (PCC), when the grid is distorted, the reference current is not generated correctly. This issue is treated in detail in [11]. A selective harmonic-compensation control is used in order to mitigate the grid voltage harmonics, but with the inevitable trade-off between filtering performance and system stability, specially for the compensation of higher order harmonics [12].

Different control strategies for active power filters have been reported in the literature, such as linear feedback control [13], nonlinear control [14], repetitive control [15], neural network approach [16], [17], and adaptive fuzzy control [18], [19]. Any of the aforementioned control methods for three-phase SAPFs have been implemented in $d q$ or $\alpha \beta$ frames. These control techniques have been widely accepted and used by researchers. Despite this, and with the aim of increasing robustness with a fast dynamic response, sliding mode control (SMC) is the best alternative to be used [20]. Here there is a lack of research using this technique due to the following drawbacks: a) the cross-coupling between controllers through the neutral point voltage which causes severe interferences between controllers [21] and b) the variable switching frequency of the SMC which 


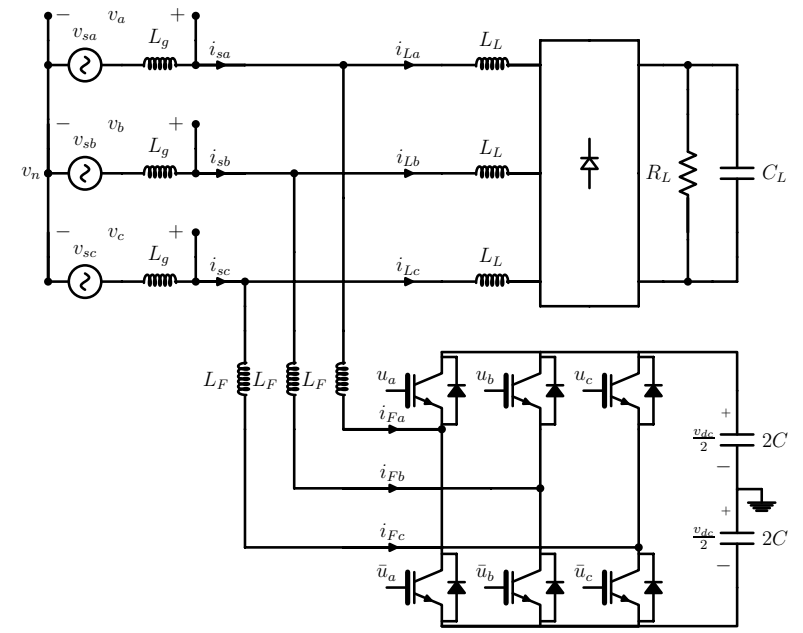

Fig. 1. Circuit diagram of three-phase SAPF

is unwanted in most industrial applications [22].

Some authors have reported several alternatives to fix the switching frequency, being the digital hysteretic modulators the most common solution [23], [24]. However, a high sampling frequency is needed for the correct implementation of these digital hysteretic modulators to increase the effectiveness of the control algorithm. This problem can be solved by using analog hysteretic comparators but at the expense of increasing the hardware [25]. Other solutions are adopted by using predictive control and DSP timers in order to decide the switching time [22]. It is worth to mention that the fixed switching frequency algorithms generally assume a decoupling between controllers, which is usually obtained by removing the neutral point voltage influence from the dynamics of the controllers. Some researchers have studied this issue but generally, their proposals have been based on a pure cancellation. At this point, it is important to highlight that the effectiveness of this cancellation has a clear dependence on the deviation of system parameters, and a high sensitivity to the system noise.

In order to avoid the aforementioned problems, a new model-based control solution is presented in this paper that uses SMC with estimated states. This solution is not usually applied to control power converters and, to our knowledge, it has been only applied to control a unity power factor rectifier [26]. Following a similar idea, this technique can be applied to control a SAPF. The proposed method is based on simple current sliding-mode controllers which use estimated variables. A lineal converter model is used in a Kalman filter (KF) in order to obtain the estimated states. This model allows a state estimation without the influence of the neutral point voltage, thus eliminating any interference between controllers in a simple and effective way. Then, these estimated states are used to obtain three independent sliding surfaces with a high robustness against variations of system parameters and noise immunity due to the application of the KF. With the proposed control method, the THD of the current delivered by the grid is improved compared with the standard SMC using measured variables. In addition, a variable hysteresis band comparator combined with a switching decision algorithm are used to concentrate the switching spectrum, allowing to operate at fixed switching frequency.

The main advantage of this control method applied to a SAPF lies in the fact that the current reference is obtained from the fundamental component of the PCC voltage. This voltage is computed using the proposed model in a KF algorithm. With this procedure, the reference current is obtained without any distortion, even in the case of a highly distorted grid, without using any specific harmonic filters. An interesting side effect of the proposed control scheme is that in the case of voltage sags, the PCC voltage and its quadrature can be obtained, and positive and negative sequences can be derived directly without using a specific PLL algorithm for voltage sequence extraction, such as the algorithm presented in [27]. As shown in the experimental results section, very satisfactory results have been obtained using this control technique.

The main contributions of this paper are: 1) a linear converter model is derived, 2) a model-based control is used for the SAPF using the proposed linear converter model, which allows to estimate the fundamental component of the voltage at the PCC even in case of distortion in the grid, and also to remove the neutral point voltage influence from the dynamics of the controllers, and 3) an improvement in the THD of the currents delivered by the grid is achieved, compared with the THD obtained when the conventional SMC is applied.

The paper is organized as follows. In section II the nonlinear model of the SAPF is presented. In section III the problem formulation is introduced. Section IV presents the control proposal. The experimental results are presented in section V. Finally, section VI draws some conclusions.

\section{Non-Linear Model of The SAPF}

Fig. 1 shows a circuit scheme of the SAPF. From this circuit the converter differential equations can be written as follows:

$$
\begin{aligned}
L_{F} \frac{d \mathbf{i}_{F}}{d t} & =\mathbf{v}-\frac{v_{d c}}{2} \mathbf{u}+v_{n} \mathbf{1} \\
C \frac{d v_{d c}}{d t} & =i_{F a} \frac{u_{a}}{2}+i_{F b} \frac{u_{b}}{2}+i_{F c} \frac{u_{c}}{2} \\
v_{n} & =\frac{v_{d c}}{6}\left(u_{a}+u_{b}+u_{c}\right)
\end{aligned}
$$

where $v_{n}$ is the neutral point voltage, $\mathbf{i}_{F}=\left[\begin{array}{lll}i_{F a} & i_{F b} & i_{F c}\end{array}\right]^{T}$ is the inductor filter current vector, $\mathbf{v}=\left[\begin{array}{lll}v_{a} & v_{b} & v_{c}\end{array}\right]^{T}$ is the voltage at the PCC $\mathbf{u}=\left[\begin{array}{lll}u_{a} & u_{b} & u_{c}\end{array}\right]^{T}$ is the control variables vector with $u_{a, b, c} \in\{ \pm 1\}, v_{d c}$ is the dc voltage at the SAPF capacitor and $\mathbf{1}$ is defined as $\left[\begin{array}{lll}1 & 1 & 1\end{array}\right]^{T}$.

In order to obtain the state-space model the equations mentioned above can be rewritten as follows:

$$
\begin{aligned}
\frac{d \mathbf{x}}{d t} & =\mathbf{f}(\mathbf{x}, \mathbf{u})+\mathbf{E}\left(\mathbf{v}+v_{n}\right) \\
\mathbf{y} & =\mathbf{h}(\mathbf{x}, \mathbf{u})
\end{aligned}
$$

where

$$
\begin{aligned}
\mathbf{x} & =\left[\begin{array}{llll}
i_{F a} & i_{F b} & i_{F c} & v_{d c}
\end{array}\right]^{T} \\
\mathbf{f}(\mathbf{x}, \mathbf{u}) & =\left(\begin{array}{cccc}
0 & 0 & 0 & -\frac{u_{a}}{2 L_{F}} \\
0 & 0 & 0 & -\frac{u_{b}}{2 L_{F}} \\
0 & 0 & 0 & -\frac{u_{c}}{2 L_{F}} \\
\frac{u_{a}}{2 C} & \frac{u_{b}}{2 C} & \frac{u_{c}}{2 C} & 0
\end{array}\right) \mathbf{x}
\end{aligned}
$$




$$
\mathbf{E}=\frac{1}{L_{F}}\left[\begin{array}{llll}
1 & 1 & 1 & 0
\end{array}\right]^{T}
$$

and the output matrix is given by $\mathbf{h}$ where is accomplished that

$$
\mathbf{H}=\frac{\partial \mathbf{h}}{\partial \mathbf{x}}=\left(\begin{array}{cccc}
1 & 0 & 0 & 0 \\
0 & 1 & 0 & 0 \\
0 & 0 & 1 & 0 \\
0 & 0 & 0 & 1
\end{array}\right)
$$

\section{PROBlem Formulation}

The main control objective in a SAPF is to guarantee sinusoidal grid currents in phase with the grid voltages in presence of nonlinear loads. As a first approach, the sliding surfaces can be generated using the grid currents vector in order to obtain a grid current in phase with the voltage source:

$$
\mathbf{S}=\mathbf{i}_{s}^{*}-\mathbf{i}_{s}
$$

where $\mathbf{i}_{s}^{*}=k \mathbf{v}=\left[\begin{array}{lll}i_{s a}^{*} & i_{s b}^{*} & i_{s c}^{*}\end{array}\right]^{T}$, is the reference current vector and $\mathbf{i}_{s}=\mathbf{i}_{F}+\mathbf{i}_{L}$ being $\mathbf{i}_{L}=\left[\begin{array}{lll}i_{L a} & i_{L b} & i_{L c}\end{array}\right]^{T}$ the load current vector.

Using (1), (3) and (10) the dynamics for each sliding surface is represented by the following expressions:

$$
\begin{aligned}
& \frac{d S_{a}}{d t}=\frac{d i_{s a}^{*}}{d t}-\frac{1}{L_{F}}\left(v_{a}+\frac{v_{o}}{2} \frac{-2 u_{a}+u_{b}+u_{c}}{3}\right)-\frac{d i_{L a}}{d t} \\
& \frac{d S_{b}}{d t}=\frac{d i_{s b}^{*}}{d t}-\frac{1}{L_{F}}\left(v_{b}+\frac{v_{o}}{2} \frac{u_{a}-2 u_{b}+u_{c}}{3}\right)-\frac{d i_{L b}}{d t} \\
& \frac{d S_{c}}{d t}=\frac{d i_{s c}^{*}}{d t}-\frac{1}{L_{F}}\left(v_{c}+\frac{v_{o}}{2} \frac{u_{a}+u_{b}-2 u_{c}}{3}\right)-\frac{d i_{L c}}{d t}
\end{aligned}
$$

The aforementioned expressions show two different issues to take into account: 1) a cross-coupling term introduced by the neutral point voltage (3) which is expressed as a linear combination of the control signals, $u_{a, b, c}$, in each sliding surface dynamics. This term can produce severe irregularities in the ordinary hysteresis operation since each phase-leg can not be controlled independently [26], and 2) the reference currents are generated using the measured PCC voltages, $i_{s a, s b, s c}^{*}=k v_{a, b, c}$, and for this reason the distortion in the grid will involve grid currents with higher harmonic content.

As a solution, this paper suggest a model-based method in which three independent sliding mode controllers will be designed while obviating the neutral point voltage in the converter model used in a KF. This fact has a negligible effect in the average dynamics of the closed loop system. In addition, the fundamental component of the voltage at the PCC is estimated in order to generate the reference currents, even in the case of a distorted grid.

An interesting side effect of the proposed control scheme is the fact that the sliding surfaces for each controller will be designed independently. This decoupling property between the three current controllers allows using a fixed switching frequency algorithm. The proposed model to achieve all this aims will be introduced in the next section.

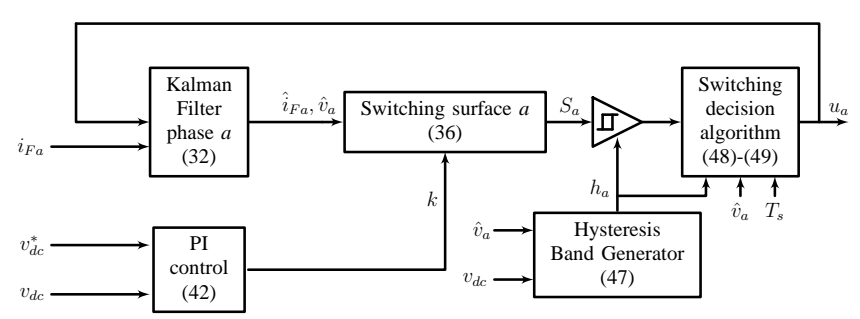

Fig. 2. Proposed control system for phase-leg $a$ of the SAPF

\section{Proposed Control System}

The control diagram for phase-leg $a$ is depicted in Fig.2. Phase-legs $b$ and $c$ have the same control scheme. A KF is used together with the proposed converter model, as explained in the next subsection, in order to estimate the states. A PI controller is used to regulate the filter output voltage and to obtain the value of the gain $k$. This gain and the state estimation are the inputs of a sliding surface which computes the error between the estimated grid current and its reference, obtained as $i_{s a}^{*}=$ $k \hat{v}_{s a}$. Finally this error is considered in a variable hysteresis comparator, which is used together with a switching decision algorithm. This combination allows to obtain a fixed switching frequency with an improved switching spectrum.

\section{A. Proposed state-space model of the converter}

The objective of this section is to obtain a linear simplified model for the SAPF in order to use it in the KF algorithm. This model will be designed with the aim of solving the problems exposed in section III. The first problem was related to the axis-decoupling and it can be solved by eliminating the dependence of the neutral point voltage from the controller dynamics (11)-(13). This is also an opportunity to reduce the number of sensors by adding two additional states, the voltage at the PCC $v_{i}$ and its quadrature $v_{i q}$, in order to estimate the fundamental component of this voltage. Due to the grid inductance and grid voltage harmonics, the measured voltage at the PCC can be distorted. Thus, this estimation will improve the quality in the generation of reference currents, leading to a reduction in the THD of the grid currents. With this approach the second problem can be solved. In addition, the estimated PCC voltage can also be used in the hysteresis band expressions explained in section V-D.

Hence, in order to derive the adequate SAPF model, some considerations must be taken into account. Firstly, is to ensure that the model is a decoupled model (i.e. each phase only depends on its own control variable) and secondly, the model must be linear, accurate, and simple to reduce the computational time. For these reasons the following assumptions are made:

1) The neutral point voltage, $v_{n}$ in the filter current equation (1) is not used in the proposed model, since it is a high frequency signal (it depends on the control actions $u_{a}, u_{b}$, $u_{c}$ ), and it only affects current inductors ripples. Thus, this approximation has a negligible effect on the average dynamics of the closed-loop system.

2) The SAPF capacitor is usually large and its voltage has a slow dynamics. Hence, the state variable $v_{d c}$ can be assumed 
to be constant between different sampling instants of the same switching period. Besides, since this variable is measured, it can be considered as a parameter and its dynamics can be neglected. With this consideration, the new state-space model for each phase-leg $i$ could be considered linear in a switching period [28].

3) The voltage at the PCC $v_{i}$ and its quadrature $v_{i q}$ are added in the model in order to estimate the fundamental component of the PCC voltages. With this solution the generation of the reference currents does not rely on noise or harmonic distortion in the grid, thus improving the system robustness against grid disturbances.

The aforementioned considerations lead to redefine new differential equations for each phase-leg $i$ :

$$
\begin{aligned}
\frac{d i_{F i}}{d t} & =\frac{1}{L_{F}} v_{i}-\frac{v_{d c}}{2 L_{F}} u_{i} \\
\frac{d v_{i}}{d t} & =\omega_{o} v_{i q} \\
\frac{d v_{i q}}{d t} & =-\omega_{o} v_{i} .
\end{aligned}
$$

where $\omega_{o}$ is the angular grid frequency.

The proposed linear converter model can be obtained from these differential equations as follows:

$$
\begin{aligned}
\frac{d \mathbf{x}_{i}}{d t} & =\mathbf{A} \mathbf{x}_{i}+\mathbf{B} v_{d c} u_{i} \\
y_{i} & =\mathbf{C} \mathbf{x}_{i}
\end{aligned}
$$

being

$$
\begin{gathered}
\mathbf{x}_{i}=\left[\begin{array}{lll}
i_{F i} & v_{i} & v_{i q}
\end{array}\right]^{T} \\
\mathbf{A}=\left(\begin{array}{ccc}
0 & \frac{1}{L_{F}} & 0 \\
0 & 0 & \omega_{o} \\
0 & -\omega_{o} & 0
\end{array}\right) \\
\mathbf{B}=\left[\begin{array}{ccc}
-\frac{1}{2 L_{F}} & 0 & 0
\end{array}\right]^{T}
\end{gathered}
$$

Using this model, a KF can be employed to estimate the filter current without the effect of the neutral point voltage and to extract the fundamental component and its quadrature of the PCC voltages. This fact will allow to generate sinusoidal reference currents even in case of a distorted grid [29].

Note that the control algorithm does not use grid voltage sensors and the value of the output matrix $\mathbf{C}$ is:

$$
\mathbf{C}=\left[\begin{array}{lll}
1 & 0 & 0
\end{array}\right]
$$

At this point it is important to prove the observability and controllability of the proposed converter model. Taking into account the aforementioned matrices, the following observability matrix is obtained

$$
\mathbf{O}=\left[\begin{array}{lll}
\mathbf{C} & \mathbf{C A} & \mathbf{C A}^{2}
\end{array}\right]^{T} .
$$

Moreover the controllability matrix $\Gamma$ is given by

$$
\boldsymbol{\Gamma}=\left[\begin{array}{lll}
\mathbf{B} & \mathbf{A B} & \mathbf{A}^{2} \mathbf{B}
\end{array}\right]
$$

Matrices $\mathbf{O}$ and $\boldsymbol{\Gamma}$ are both of full-rank, (i.e. $\operatorname{rank}\{\mathbf{O}\}=$ $\operatorname{rank}\{\boldsymbol{\Gamma}\}=3$ ), so that the system is controllable and can be observed using only the measured filter current $i_{F i}$.

\section{B. Kalman Filter}

It is well known that in nonlinear system applications an EKF is used in order to estimate the states, with the drawback of the long computational time required by this algorithm. This is the case of the SAPF which is clearly a nonlinear system (4)-(5). However, the proposed converter model defined by (17)-(18) is considered linear in a switching period, and it can be used for each phase-leg $i$ in order to estimate the states. This fact allows to use a KF instead of an EKF, which reduces the computational load. For the digital KF implementation, the state-space model (17)-(18) is discretized, yielding

$$
\begin{aligned}
\mathbf{x}_{i}(k+1) & =\mathbf{A}_{d} \mathbf{x}_{i}(k)+\mathbf{B}_{d} v_{d c}(k) u_{i}(k)+\boldsymbol{\eta}_{i}(k) \\
y_{i}(k) & =\mathbf{C x}_{i}(k)+\mathbf{w}_{i}(k)
\end{aligned}
$$

where $\boldsymbol{\eta}_{i}(k)$ and $\mathbf{w}_{i}(k)$ are the process and measurement noise vectors respectively; their covariance matrices are given by:

$$
\begin{aligned}
& \mathbf{R}_{i}(k)=E\left\{\mathbf{w}_{i}(k) \mathbf{w}_{i}^{T}(k)\right\} \\
& \mathbf{Q}_{i}(k)=E\left\{\boldsymbol{\eta}_{i}(k) \boldsymbol{\eta}_{i}^{T}(k)\right\}
\end{aligned}
$$

and

$$
\begin{gathered}
\mathbf{A}_{d}=e^{A T_{s}} \cong \mathbf{I}+\mathbf{A} T_{s}=\left(\begin{array}{ccc}
1 & \frac{T_{s}}{L} & 0 \\
0 & 1 & \omega_{o} T_{s} \\
0 & -\omega_{o} T_{s} & 1
\end{array}\right) \\
\mathbf{B}_{d}=\int_{0}^{T_{s}} B e^{A \lambda} d \lambda \cong \mathbf{B} T_{s}=\left[\begin{array}{ccc}
-\frac{T_{s}}{2 L} & 0 & 0
\end{array}\right]^{T}
\end{gathered}
$$

being $\mathbf{I}$ the identity matrix and $T_{s}$ the sampling time.

Here it is assumed that $v_{d c}(k)$ is a constant between samples of the same switching period, so that $v_{d c}(k+1) \cong v_{d c}(k)$, and the discrete model can be considered linear over the same switching period.

As mentioned above, the state-space model defined by (26)(27) is used in a KF, and the estimated states can be used as inputs of the sliding surfaces providing three independent controllers with a high noise immunity and an improved THD of the currents injected to the grid. The main properties and the implementation of the KF are well explained in [30] so only the adaptive equation used in the estimation will be given in this section.

Based on the KF algorithm implementation, the equation for the state estimation can be expressed as follows:

$$
\begin{aligned}
\hat{\mathbf{x}}_{i}(k+1) & =\mathbf{A}_{d} \mathbf{x}_{i}(k)+\mathbf{B}_{d} v_{d c}(k) u_{i}(k) \\
& +\mathbf{L}_{i}(k)\left(\mathbf{x}_{i}(k)-\hat{\mathbf{x}}_{i}(k)\right)
\end{aligned}
$$

where the Kalman gain is computed as:

$$
\mathbf{L}_{i}(k)=\mathbf{P}_{i}(k) \mathbf{C}^{T}\left(\mathbf{C P}_{i}(k) \mathbf{C}^{T}+\mathbf{R}_{i}(k)\right)^{-1} .
$$

being $\mathbf{P}_{i}$ the error covariance matrix for phase-leg $i$, which is computed following the KF algorithm explained in [26].

1) Considerations about the Kalman gain computation: The high computational load is a major drawback in the KF algorithm implementation. Particularly, the Kalman gain (33) contains a matrix inversion which involves a long computational time. Using a KF for each phase-leg instead a single $\mathrm{KF}$ for the whole three-phase system, the matrix inversion in (33) can be reduced to a scalar inversion. Actually, according 
to (23) and with the condition that $\mathbf{P}_{i}(k)$ is a square matrix, the computation of $\mathbf{C P}_{i}(k) \mathbf{C}^{T}$ in the Kalman gain expression results in a scalar quantity. Then, to be consistent with the matrix addition operation in (33), the noise covariance matrix $\mathbf{R}_{i}(k)$ is considered as mean noise power in each phase leg, $R_{i}(k)$. In addition, considering that this noise is so similar in each phase leg, the Kalman gain can be assumed identical for all the phases (i.e. $\mathbf{L}_{a}(k) \cong \mathbf{L}_{b}(k) \cong \mathbf{L}_{c}(k)$ ). Thanks to this approximation, (33) is computed only for one phase and it is used in all the phases. Following this approach a notably reduction in the KF implementation is practically obtained.

2) Mean noise power estimation: The value of $R_{i}(k)$ can be estimated using the unbiased mean power estimator

$$
R_{i}(k)=\frac{1}{N T_{s}} \sum_{k=0}^{N-1}\left|w_{i}(k)\right|^{2}
$$

where $w_{i}(k)$ is an additive white gaussian noise sample at time $k$, in phase-leg $i$, and $N$ is the number of samples.

To obtain $w_{i}(k)$, the simplest way is to use a dc-voltage with additive white gaussian noise. In this case a reference dcvoltage used in the sensing system $V_{\text {ref }}$ has been employed. This voltage is connected to one analog input of the DSP and is contaminated by the switching noise when the inverter is switching. The noise samples can be obtained by subtracting from the $V_{\text {ref }}$ measures, the real value, $V_{r e f}^{*}$ (i.e. the measured value when the inverter is not switching), yielding

$$
R_{i}(k)=\frac{1}{N T_{s}} \sum_{k=0}^{N-1}\left|V_{r e f}(k)-V_{r e f}^{*}(k)\right|^{2}
$$

The last expression allows to estimate the mean noise power in any of the three phases and it can be used to compute the Kalman gain. Assuming the noise power level has no important variations, $R_{i}(k)$ is computed at the beginning, remaining unchanged during all the time.

3) Process covariance matrix: Matrix $\mathbf{Q}_{i}(k)$ is not found so directly. It is generally derived intuitively, but there are some points that need to be regarded in its selection [30]. Unmodeled dynamics and parameter uncertainties are generally modeled as process noise. Usually $\mathbf{Q}_{i}(k)$ is taken as a diagonal matrix, which is of dimension 3 in this application. Its value has been obtained by means of simulations. In the simulation results an approximate value of $\mathbf{Q}_{i}(k)=0.005 \mathbf{I}_{3}$ provides an adequate behaviour, where $\mathbf{I}_{3}$ is a 3 -dimensional unity matrix.

\section{Sliding-Mode Control}

The conventional SAPF control scheme consists of a fastinner input-current loop which ensures sinusoidal input currents in phase with the PCC voltages, $\mathbf{i}_{s}=k \mathbf{v}$, and a slowouter loop, usually a PI controller, the main task of which is to regulate the output voltage, modifying the input-current amplitudes. In this proposal, the inner control loop is designed using estimated variables as explained in previous sections. With this aim the following switching surfaces are proposed:

$$
\begin{aligned}
S_{a} & =\hat{i}_{s a}^{*}-\hat{i}_{s a} \\
S_{b} & =\hat{i}_{s b}^{*}-\hat{i}_{s b} \\
S_{c} & =\hat{i}_{s c}^{*}-\hat{i}_{s c} .
\end{aligned}
$$

It should be noticed that using our proposed model, the dynamics of the aforementioned controllers only depends on its control variables $u_{a}, u_{b}$ and $u_{c}$ :

$$
\begin{aligned}
& \frac{d S_{a}}{d t}=\frac{d \hat{i}_{s a}^{*}}{d t}-\frac{1}{L_{F}}\left(\hat{v}_{a}-\frac{v_{d c}}{2} u_{a}\right)-\frac{d i_{L a}}{d t} \\
& \frac{d S_{b}}{d t}=\frac{d \hat{i}_{s b}^{*}}{d t}-\frac{1}{L_{F}}\left(\hat{v}_{b}-\frac{v_{d c}}{2} u_{b}\right)-\frac{d i_{L b}}{d t} \\
& \frac{d S_{c}}{d t}=\frac{d \hat{i}_{s c}^{*}}{d t}-\frac{1}{L_{F}}\left(\hat{v}_{c}-\frac{v_{d c}}{2} u_{c}\right)-\frac{d i_{L c}}{d t}
\end{aligned}
$$

where $\hat{i}_{s a, s b, s c}^{*}=k \hat{v}_{a, b, c}$ are the reference currents using the estimated inductor filter currents, $\hat{i}_{s a, s b, s c}=\hat{i}_{F a, F b, F c}+$ $i_{L a, L b, L c}$ are the estimated grid currents and $k$ is implemented in the outer control loop in order to regulate the output capacitor voltage using a PI controller [5], and expressed as:

$$
k=k_{p}\left(v_{d c}^{*}-v_{d c}\right)+k_{i} \int_{-\infty}^{t}\left(v_{d c}^{*}-v_{d c}\right) d \tau
$$

being $k_{p}$ and $k_{i}$ the proportional and integral gains respectively.

The main requirement in the design of the SMC is to satisfy the reaching conditions, and also to guarantee the existence of a sliding regime in the switching surfaces $\mathbf{S}=0$. The most often used reaching conditions for each phase-leg $i$ are given by

$$
S_{i} \dot{S}_{i}<0
$$

Defining the control variable $u_{i}$ as:

$$
u_{i}=\left\{\begin{array}{lll}
u_{i}^{+} & \text {if } & S_{i}>0 \\
u_{i}^{-} & \text {if } & S_{i}<0
\end{array}\right.
$$

where $u_{i} \in\{1,-1\}$, and taking into account (43), (14) and (36)-(38) the following expression can be deduced:

$$
\frac{v_{d c}}{2 L_{F}}\left(u_{i}^{+}-u_{i}^{-}\right)<0
$$

which allows us to determine the switching action

$$
u_{i}=\left\{\begin{array}{lll}
u_{i}^{+}=-1 & \text { if } & S_{i}>0 \\
u_{i}^{-}=1 & \text { if } & S_{i}<0
\end{array}\right.
$$

\section{Hysteresis Band Generator}

This section deals with a fully digital hysteretic modulator to fix switching frequency of the SAPF. Conventionally, the expression for the hysteresis band is given by the following equation [25]

$$
h_{i}=\frac{v_{o}}{8 L_{F} f_{s w}}\left[1-\left(\frac{2 \hat{v}_{i}}{v_{d c}}\right)^{2}\right]
$$

where $f_{s w}$ is the desired switching frequency. 


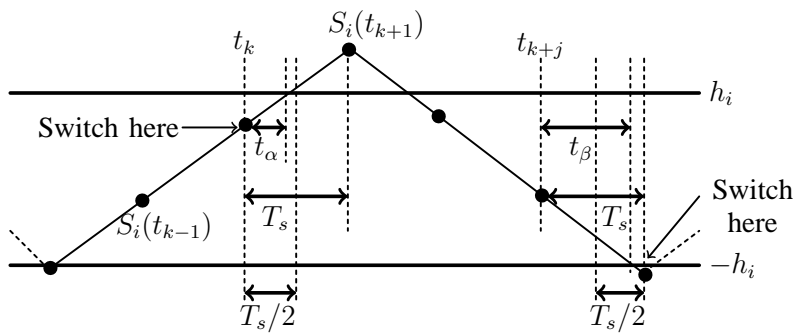

Fig. 3. Hysteresis band with the switching surface.

It is widely known that the finite sampling frequency can cause deviations in the desired the switching frequency due to the samples that appear out of the hysteresis limits. This problem is shown in Fig. 3 and it can be avoided by using a simple switching decision algorithm presented in [26]. This algorithm, which has been adopted for this application, can decide the optimum switching instant and it concentrates the switching spectrum. Using this algorithm, the number of samples out of the hysteresis limits is drastically reduced. Assuming a slow variation in the hysteretic band in a sampling period, $h_{i}\left(t_{k}\right) \simeq h_{i}\left(t_{k+1}\right)$, the algorithm can be implemented as follows: 1) When a sample is acquired, the time between the sample and the hysteresis band is calculated. If the sample is in the increasing slope of the switching surface, $\left(u_{i}=1\right)$ the time $t_{\alpha}$ can be computed as:

$$
t_{\alpha} \cong L_{F} \frac{h_{i}-S_{i}\left(t_{k}\right)}{v_{d c} / 2-\hat{v}_{i}}
$$

otherwise, if the sample is in the decreasing slope of the switching surface $\left(u_{i}=-1\right)$, the time $t_{\beta}$ can be approximated by:

$$
t_{\beta} \cong L_{F} \frac{h_{i}+S_{i}\left(t_{k+j}\right)}{v_{d c} / 2+\hat{v}_{i}}
$$

being $S_{i}$ the switching surface for the phase-leg $i$.

2) When the estimated times $t_{\alpha}$ and $t_{\beta}$ are known, the switching decision is implemented as follows:

In the case of a positive slope:

- If $t_{\alpha}<\frac{T_{s}}{2}$, the control signal $u_{i}$ is changed from 1 to -1 , otherwise the control signal $u_{i}$ is not changed.

In the case of a negative slope:

- If $t_{\beta}<\frac{T_{s}}{2}$, the control signal $u_{i}$ is changed from -1 to 1 , otherwise the control signal $u_{i}$ is not changed.

This procedure is done for each sampling period.

\section{EXPERIMENTAL RESULTS}

Fig. 4 shows the prototype of the SAPF built using a 4.5kVA SEMIKRON full-bridge as the power converter and a TMS320F28M36 floating-point digital signal processor (DSP) as the control platform. The grid voltages have been generated using a PACIFIC 360-AMX source. Some experimental results have been exported to MATLAB (C) for the final representation. The system parameters are listed in Table I.

Using the approximations reported in section IV-B, the computational load of the KF algorithm can be reduced from $12 \mu \mathrm{s}$ to $3 \mu \mathrm{s}$. Note that in each phase-leg a different KF is used
TABLE I

SYSTEM PARAMETERS

\begin{tabular}{ccc}
\hline \hline Symbol & Description & Value \\
\hline$L_{F}$ & Filter input inductance & $5 \mathrm{mH}$ \\
$C$ & Output capacitor & $1500 \mu \mathrm{F}$ \\
$v_{d c}$ & dc voltage & $400 \mathrm{~V}$ \\
$f_{s}$ & Sampling frequency & $40 \mathrm{kHz}$ \\
$f_{s w}$ & Switching frequency & $4 \mathrm{kHz}$ \\
$f_{g r i d}$ & Grid frequency & $60 \mathrm{~Hz}$ \\
$V_{g r i d}$ & Grid voltage & $110 \mathrm{Vrms}$ \\
$L_{g}$ & Grid inductance & $0.5 \mathrm{mH}$ \\
$k_{p}$ & Proportional gain & 0.03 \\
$k_{i}$ & Integral gain & 0.5 \\
$R_{L}$ & Load resistor & $48 \Omega-24 \Omega$ \\
$L_{L}$ & Load inductance & $5 \mathrm{mH}$ \\
$C_{L}$ & Load capacitor & $100 \mu \mathrm{F}$ \\
$R_{i}(k)$ & Single phase system noise power & $0.24 V^{2}$ \\
$Q_{i}(k)$ & Process covariance matrix & $0.005 \mathbf{I}_{3}$ \\
\hline
\end{tabular}

but the Kalman gain is the same for each phase-leg. This is an important reduction in the total algorithm time, which now is about $23 \mu \mathrm{s}$. This fact allows a sampling frequency $f_{s}=40 \mathrm{kHz}$ and it makes possible the implementation of this proposal in the DSP. The switching frequency, $f_{s w}$, when a sliding mode control is used can be chosen as $f_{s w}=f_{s} / 10$ to ensure enough samples for a proper performance of the variable hysteresis comparator. This relation provides a good trade-off between the use of the computational load in a switching period and the switching frequency accuracy. The following figures show the good performance of the proposed control system.

In Fig.5(a) the sliding surfaces with its corresponding control signals in the case of coupled controllers (10) are shown. In this case the three controllers are implemented with measured variables instead of estimated ones. The figure shows the effect of the neutral point voltage (3) in the dynamics of the controllers. As it can be seen, the slope of each sliding surface changes with any of the control signals losing the synchronization and making impossible to design a current control with variable hysteresis band operating at fixed switching frequency.

In Fig.5(b) the sliding surfaces with its corresponding control signals in the case of decoupled controllers (36)-(38) are shown. In this case the sliding mode controllers use the estimated estates provided by (32). As it can be seen, the axisdecoupling is effective, and the slope of each sliding surface only changes with its control variable. Using the proposed model the three controllers are independent and the variable hysteresis control can be performed ensuring a fixed switching frequency.

\section{A. Response of the SAPF to load variations}

Fig.6 represents the main waveforms of the SAPF when a sudden load step change occurs, from no load to full-load, and from full-load to half-load. This figure shows from top to bottom the grid currents, the non-linear load currents, the filter currents and the output voltage. Fig.6(a) shows the waveforms when the conventional SMC is used, while the waveforms in Fig.6(b) are obtained using the proposed control scheme. Although in both figures the transient response is similar, it 


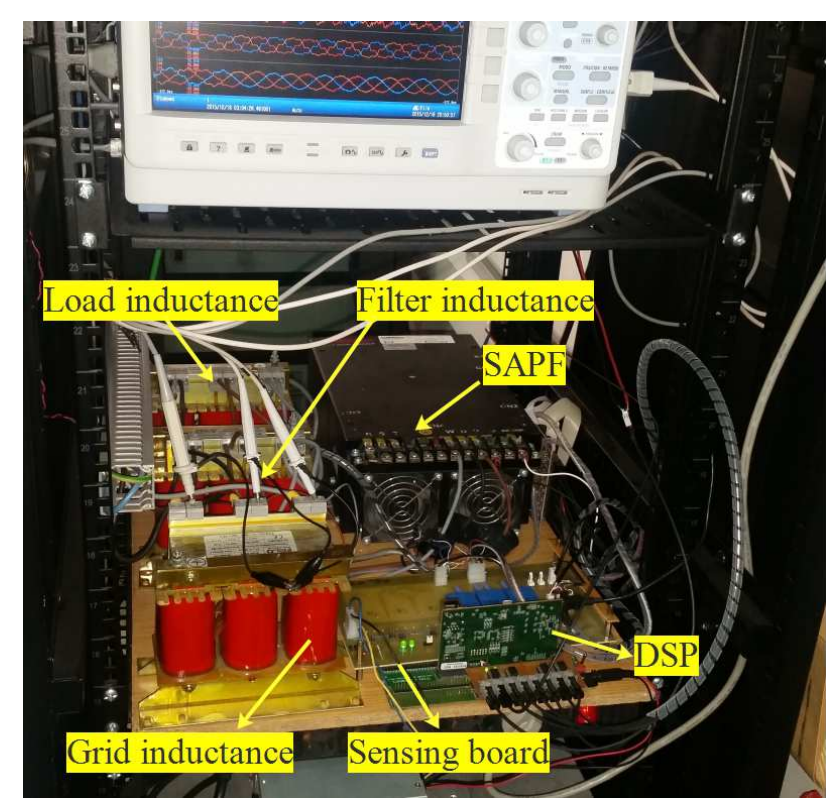

Fig. 4. SAPF Prototype

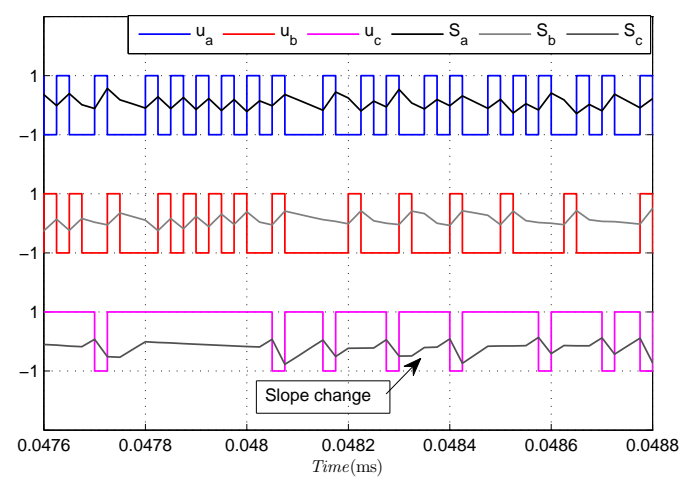

(a)

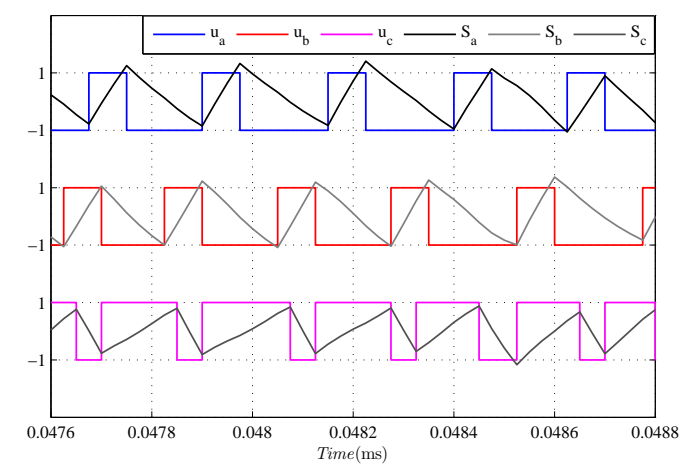

(b)

Fig. 5. Experimental control signals $u_{a}, u_{b}$ and $u_{c}$ with their corresponding sliding surfaces $S_{a}, S_{b}$ and $S_{c}$ (a) with coupled controllers, (b) with decoupled controllers operating at fixed switching frequency. can be observed that when the proposed controller is used, the THD of the grid currents is improved.

Fig.7 shows the harmonic spectrum of the grid current for phase-leg $a$. The spectrum before compensation is shown in Fig.7 $(a)$, its THD is $28.45 \%$. When the conventional SMC (10) is used, the THD is reduced to $5.36 \%$ as shown in Fig.7(b). In contrast, if the proposed controller (36)-(38) is employed, the THD is reduced to $2.51 \%$ as shown in Fig. $7(c)$. A reduction of $46.8 \%$ in the THD is achieved with respect to the conventional SMC, which proves the effectiveness of the proposed control method. Note that in both cases the fundamental component is not represented in order to have a higher resolution in the rest of the harmonics.

\section{B. SAPF performances under a distorted grid}

Fig. 8 compares the three-phase grid currents using a conventional SMC and the proposed one, in the case of a distorted grid. This figure illustrates from top to bottom: the distorted PCC voltages, being the THD about $14 \%$, the load currents, and the grid currents. As it can be seen, when the conventional SMC is used from 0 to $50 \mathrm{~ms}$, the grid currents are distorted since the reference current generation method uses distorted PCC voltages. In contrast, when the proposed controller is used from $50 \mathrm{~ms}$ to $100 \mathrm{~ms}$, the grid currents are practically sinusoidal since the reference current is generated by using only the fundamental component of the PCC voltage obtained from the KF.

A detail of the distorted PCC voltage for phase-leg $a$ together with the estimated fundamental component is shown in Fig.9. Using the estimated voltage at the PCC the reference current is sinusoidal. In addition, the estimated voltage is perfectly in phase with the measured one showing a good synchronization, at least as good as when using PLL.

\section{SAPF performances under grid voltage sags}

Fig.10 shows SAPF performances under grid voltage sags. Two different sag types are analyzed in this test: a onephase fault and a two-phase fault which are represented in Fig.10(a) and Fig.10(b), respectively. In both cases, the reference currents are obtained using the positive sequence of the PCC voltage using $i_{s a, s b, s c}^{*}=k v_{a, b, c}^{+}$. The positive sequence is computed from the estimated PCC voltages and their quadratures obtained from the KF. With this solution, the use of a specific PLL algorithm for extracting the positive and negative sequence grid voltage components is not necessary. Even without using this algorithm, the quality of the grid current waveforms are good both in normal and abnormal (voltage sag) conditions. Due to the fact that the grid current tracks only the positive sequence of the PCC voltage, a ripple frequency component of $2 \omega_{o}$ appears in the output voltage as expected.

\section{Control signal frequency spectrum}

The hysteresis band with its switching surface of phaseleg $a$ is shown in the following figures. If an hysteresis band without the switching decision algorithm is used, an error 


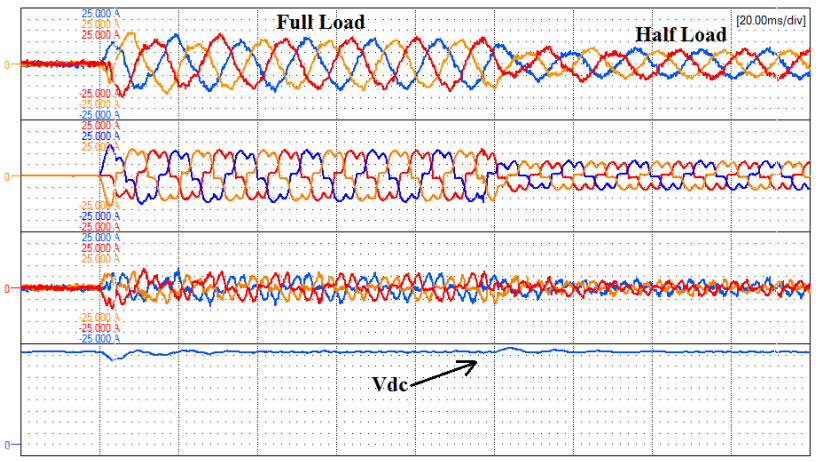

(a)

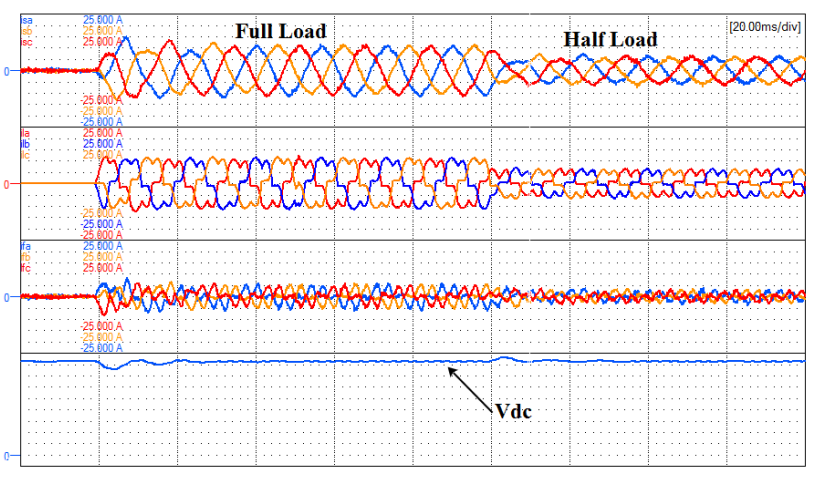

(b)

Fig. 6. A sudden load step change from no-load to full-load and full-load to half-load. From top to bottom, grid currents (5A/div), load currents $(5 \mathrm{~A} / \mathrm{div})$, filter currents (5A/div), output voltage (50V/div): a) using conventional SMC, and b) using proposed SMC.

on the desired switching frequency appears. In Fig.12(a) this problem can be clearly seen, where several samples are out of the hysteresis limits. The problem is solved using the switching decision algorithm presented in section V-C. Fig.11(b) shows how the number of samples that are out of the bounds of the hysteresis limits have been reduced using this algorithm, and the error has disappeared.

Finally, the spectrum of the switching frequency is shown in the following figures. Fig.12(a) shows the switching spectrum with hysteresis bands but without the switching decision algorithm. The spectrum is concentrated around a fixed switching frequency of $3 \mathrm{kHz}$ with an error around $1 \mathrm{kHz}$, since the desired switching frequency is $4 \mathrm{kHz}$. This problem is solved using the proposed algorithm. Its results are depicted on Fig.12(b). Now the switching frequency is the desired one, $4 \mathrm{kHz}$.

\section{CONCLUSIONS}

This paper has presented a model-based control algorithm for a three-phase SAPF. We have chosen a model-based solution using SMC in natural frame that is not usually employed in the control of power converters. The control algorithm works with a SMC which uses estimated variables from a KF achieving a high PF and reducing the THD. Besides, the use of a Kalman filter instead of a non-adaptive state observer improves the quality of the estimated signals in presence of noise, and increasing the noise immunity of the control

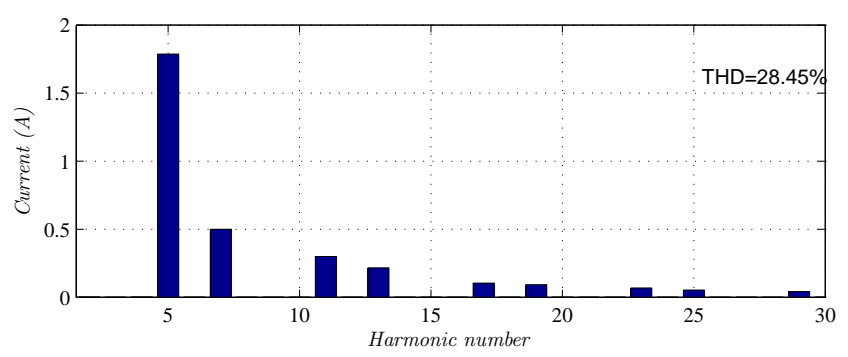

(a)

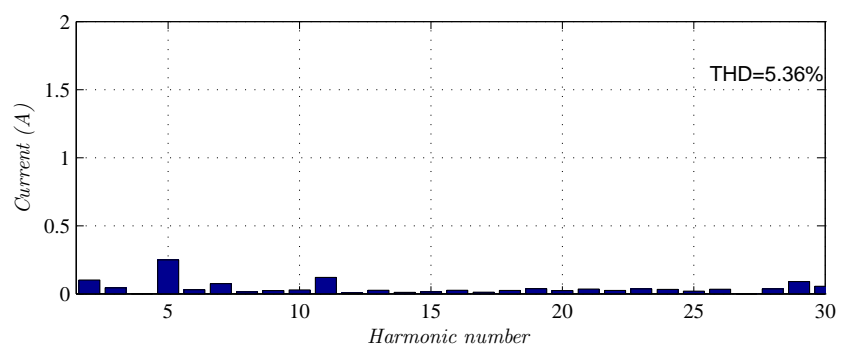

(b)

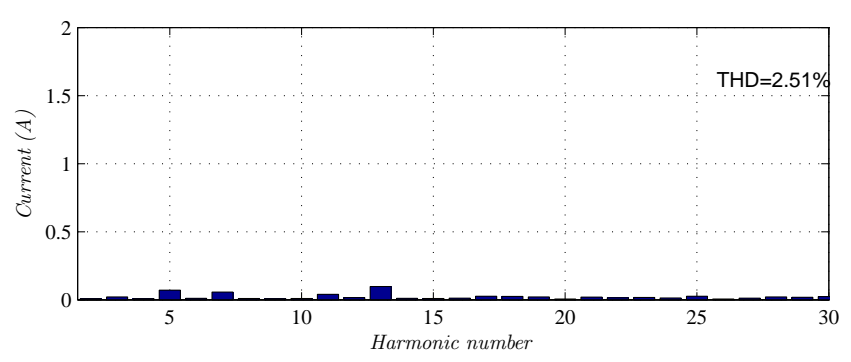

(c)

Fig. 7. Grid current harmonics for phase-leg $a$ : (a) Before compensation, (b) after compensation but using conventional SMC and (c) after compensation but using the proposed control.

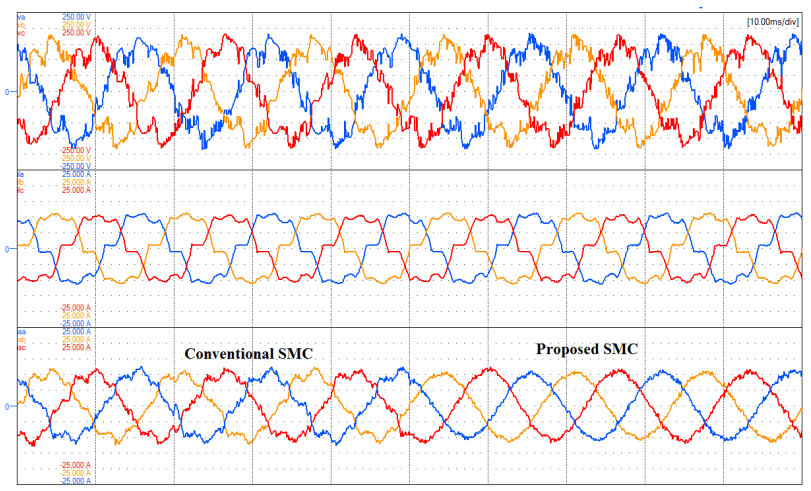

Fig. 8. From top to bottom: PCC voltages (50V/div) with $\mathrm{THD}=14 \%$, load current $(5 \mathrm{~A} / \mathrm{div})$ and grid currents $(5 \mathrm{~A} / \mathrm{div})$ 


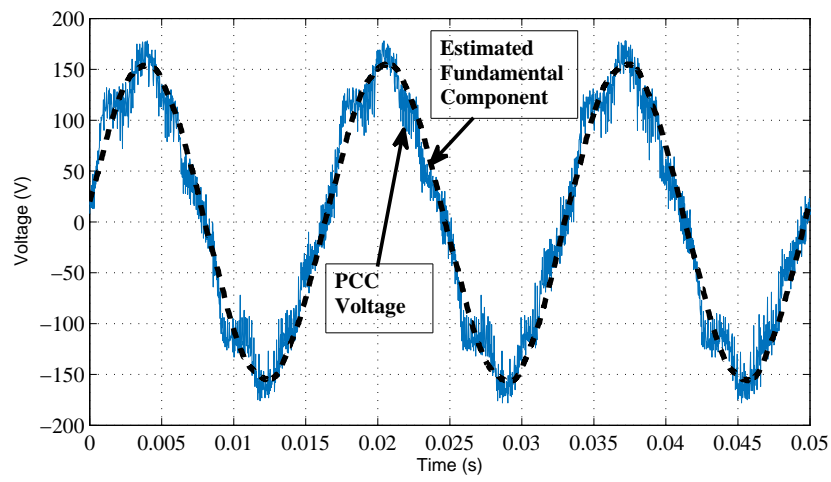

Fig. 9. Measured PCC voltage and estimated PCC voltage for phase-leg $a$.

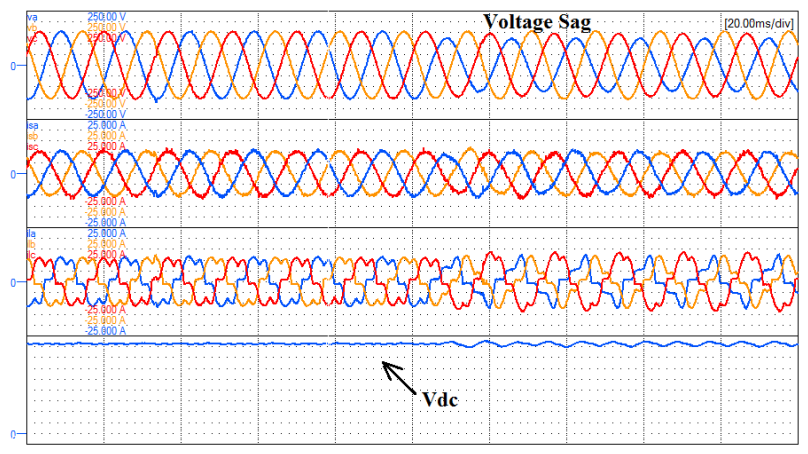

(a)

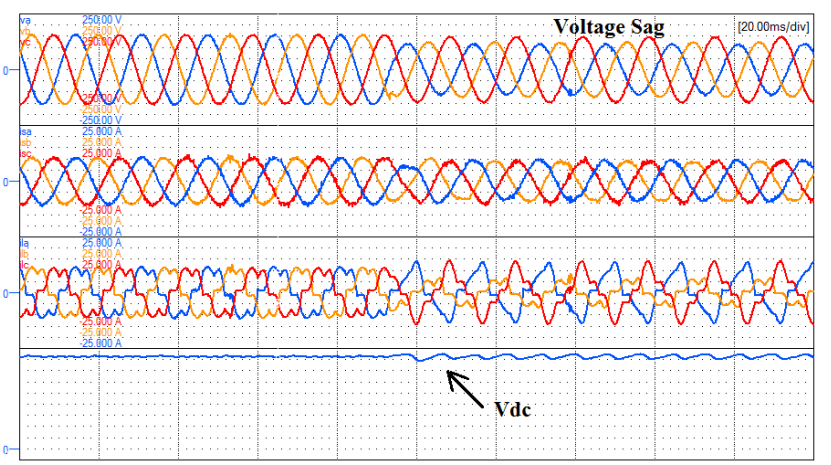

(b)

Fig. 10. From top to bottom: grid voltages (50V/div), grid currents (5A/div), load currents $(5 \mathrm{~A} / \mathrm{div})$ and $v_{d c}(50 \mathrm{~V} / \mathrm{div})$ under unbalanced grid fault: a) onephase fault and b) two-phase fault.

loop. A useful approximation has been adopted to reduce the computational time needed for the KF implementation, reducing the total time employed for the control algorithm. In addition, a fixed switching frequency algorithm has been used to improve the switching spectrum of the controllers. The corresponding experimental results show the validity of this proposal. In the near future, this technique may be applied to other converters using the most appropriate converter for each model.

\section{REFERENCES}

[1] C.-J. Wu, J.-C. Chiang, S.-S. Yen, C.-J. Liao, J.-S. Yang, and T.-Y. Guo, "Investigation and mitigation of harmonic amplification problems caused

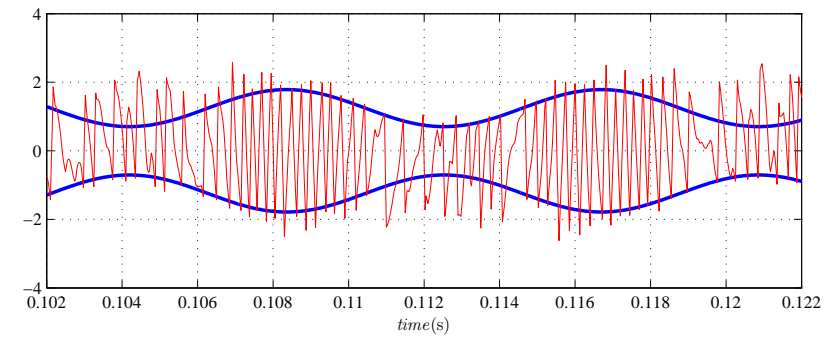

(a)

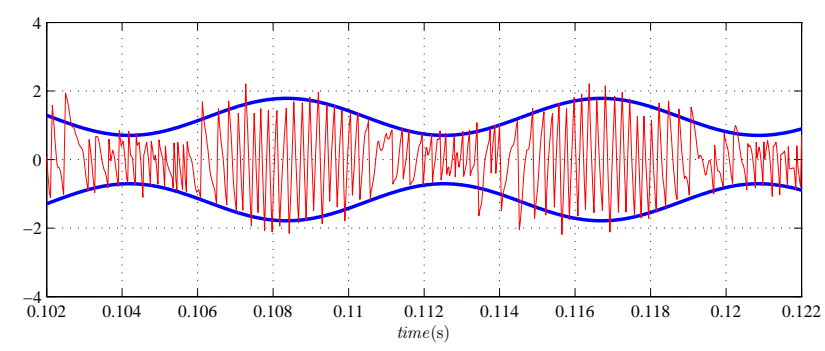

(b)

Fig. 11. Experimental hysteresis band and its switching surface for phase-leg $a$ : a) without switching decision algorithm and b) with switching decision algorithm.

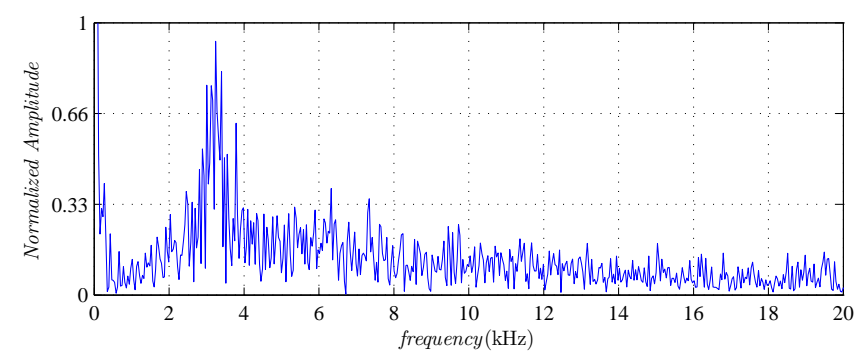

(a)

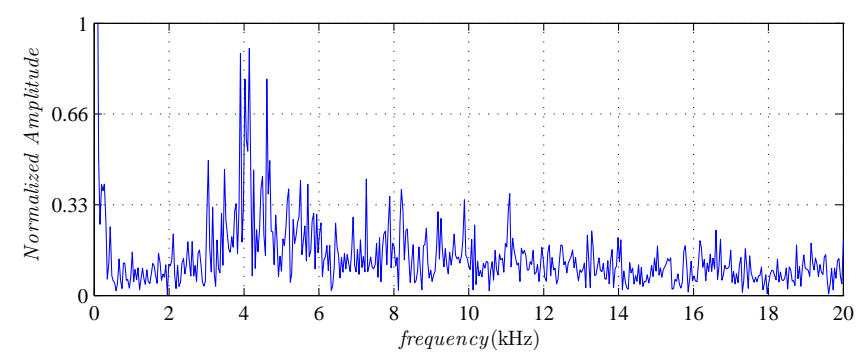

(b)

Fig. 12. switching spectrum: a) without switching decision algorithm and b) with switching decision algorithm.

by single-tuned filters," IEEE Trans. Power Delivery, vol. 13, no. 3, pp. 800-806, Jul 1998.

[2] A. Hamadi, S. Rahmani, and K. Al-Haddad, "A hybrid passive filter configuration for var control and harmonic compensation," IEEE Trans. Ind. Electron., vol. 57, no. 7, pp. 2419-2434, July 2010.

[3] L. Asiminoaei, P. Rodriguez, F. Blaabjerg, and M. Malinowski, "Reduction of switching losses in active power filters with a new generalized discontinuous-PWM strategy," IEEE Trans. Ind. Electron., vol. 55, no. 1, pp. 467-471, Jan 2008.

[4] Q.-N. Trinh and H.-H. Lee, "An advanced current control strategy for three-phase shunt active power filters," IEEE Trans. Ind. Electron., vol. 60, no. 12, pp. 5400-5410, Dec 2013.

[5] S. Rahmani, A. Hamadi, and K. Al-Haddad, "A Lyapunov-FunctionBased control for a three-phase shunt hybrid active filter," IEEE Trans. 
Ind. Electron., vol. 59, no. 3, pp. 1418-1429, March 2012.

[6] S. Rahmani, A. Hamadi, N. Mendalek, and K. Al-Haddad, "A new control technique for three-phase shunt hybrid power filter," IEEE Trans. Ind. Electron., vol. 56, no. 8, pp. 2904-2915, Aug 2009.

[7] A. Luo, C. Tang, Z. K. Shuai, W. Zhao, F. Rong, and K. Zhou, "A novel three-phase hybrid active power filter with a series resonance circuit tuned at the fundamental frequency," IEEE Trans. Ind. Electron., vol. 56, no. 7, pp. 2431-2440, July 2009.

[8] D. Yazdani, A. Bakhshai, G. Joos, and M. Mojiri, "A real-time threephase selective-harmonic-extraction approach for grid-connected converters," IEEE Trans. Ind. Electron., vol. 56, no. 10, pp. 4097-4106, Oct 2009.

[9] S. Mariethoz and A. Rufer, "Open loop and closed loop spectral frequency active filtering," IEEE Trans. Power Electron., vol. 17, no. 4, pp. 564-573, Jul 2002.

[10] V. Corasaniti, M. Barbieri, P. Arnera, and M. Valla, "Hybrid active filter for reactive and harmonics compensation in a distribution network," IEEE Trans. Ind. Electron., vol. 56, no. 3, pp. 670-677, March 2009.

[11] J. Miret, M. Castilla, J. Matas, J. Guerrero, and J. Vasquez, "Selective harmonic-compensation control for single-phase active power filter with high harmonic rejection," IEEE Trans. Ind. Electron., vol. 56, no. 8, pp. 3117-3127, Aug 2009.

[12] H. Yi, F. Zhuo, Y. Zhang, Y. Li, W. Zhan, W. Chen, and J. Liu, "A source-current-detected shunt active power filter control scheme based on vector resonant controller," IEEE Trans. Ind. Applic., vol. 50, no. 3, pp. 1953-1965, May 2014.

[13] A. Luo, X. Xu, H. Fang, H. Fang, J. Wu, and C. Wu, "Feedbackfeedforward PI-Type iterative learning control strategy for hybrid active power filter with injection circuit," IEEE Trans. Ind. Electron., vol. 57, no. 11 , pp. 3767-3779, Nov 2010.

[14] S. Rahmani, N. Mendalek, and K. Al-Haddad, "Experimental design of a nonlinear control technique for three-phase shunt active power filter," IEEE Trans. Ind. Electron., vol. 57, no. 10, pp. 3364-3375, Oct 2010.

[15] G. Escobar, P. Hernandez-Briones, P. Martinez, M. Hernandez-Gomez, and R. Torres-Olguin, "A repetitive-based controller for the compensation of $6 \ell \pm 1$ harmonic components," IEEE Trans. Ind. Electron., vol. 55, no. 8, pp. 3150-3158, Aug 2008.

[16] Y. Fang, J. Fei, and K. Ma, "Model reference adaptive sliding mode control using $r b f$ neural network for active power filter," in International Journal of Electrical Power and Energy Systems, vol. 73, June 2015, p. 249258.

[17] M. Radzi and N. Rahim, "Neural network and bandless hysteresis approach to control switched capacitor active power filter for reduction of harmonics," IEEE Trans. Ind. Electron., vol. 56, no. 5, pp. 1477-1484, May 2009.

[18] K. Ma and J. Fei, "Model reference adaptive fuzzy control of a shunt active power filter," in Ind. Electron. and Appl. (ICIEA), 2014 IEEE 9th Conference on, vol. 28, no. 1, June 2014, pp. 485-494.

[19] J. Fei and S. Hou, "Adaptive fuzzy control with fuzzy sliding switching for active power filter," in Trans. of the Institute of Measurement and Control, vol. 35, no. 8, June 2013, pp. 1094-1103.

[20] J. Matas, L. de Vicuna, J. Miret, J. Guerrero, and M. Castilla, "Feedback linearization of a single-phase active power filter via sliding mode control," IEEE Trans. Power Electron., vol. 23, no. 1, pp. 116-125, Jan 2008.

[21] L. Malesani, P. Mattavelli, and P. Tomasin, "Improved constantfrequency hysteresis current control of vsi inverters with simple feedforward bandwidth prediction," IEEE Trans. Ind. Applications., vol. 33, no. 5, pp. 1194-1202, Sep 1997.

[22] W. Stefanutti and P. Mattavelli, "Fully digital hysteresis modulation with switching-time prediction," IEEE Trans. Ind. Appl., vol. 42, no. 3, pp. 763-769, May 2006.

[23] A. Maswood and F. Liu, "A unity power factor front-end rectifier with hysteresis current control," IEEE Trans. Energy Conv., vol. 21, no. 1, pp. 69-76, March 2006.

[24] A. Maswood, E. Al-Ammar, and F. Liu, "Average and hysteresis currentcontrolled three-phase three-level unity power factor rectifier operation and performance," IET Power Electron., vol. 4, no. 7, pp. 752-758, Aug 2011 .

[25] D. Holmes, R. Davoodnezhad, and B. McGrath, "An improved threephase variable-band hysteresis current regulator," IEEE Trans. Power Electron., vol. 28, no. 1, pp. 441-450, Jan 2013.

[26] R. Guzman, L. Garcia de Vicuna, J. Morales, M. Castilla, and J. Matas, "Sliding-mode control for a three-phase unity power factor rectifier operating at fixed switching frequency," IEEE Trans. Power Electron. in press, 2015.
[27] P. Rodriguez, A. Luna, I. Candela, R. Mujal, R. Teodorescu, and F. Blaabjerg, "Multiresonant frequency-locked loop for grid synchronization of power converters under distorted grid conditions," IEEE Trans. Power Electron., vol. 58, no. 1, pp. 127-138, Jan 2011.

[28] S. Mariethoz and M. Morari, "Explicit model-predictive control of a pwm inverter with an LCL filter," IEEE Trans. Ind. Electron., vol. 56, no. 2, pp. 389-399, Feb 2009.

[29] J. Kanieski, R. Cardoso, H. Pinheiro, and H. Grundling, "Kalman filterbased control system for power quality conditioning devices," IEEE Trans. Ind. Electron., vol. 60, no. 11, pp. 5214-5227, Nov 2013.

[30] G. F. Franklin, J. D. Powell, and M. L. Workman, Digital Control of Dynamic Systems. Addison-Wesley, 1997.

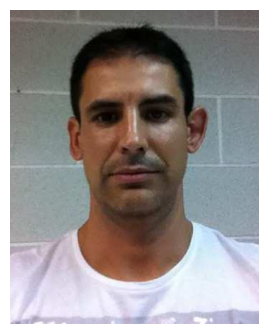

Ramon Guzman received the B.S. and M.S. degrees in telecommunications engineering from the Technical University of Catalonia, Barcelona, Spain, in 1999 and 2004, respectively. He is currently working toward the Ph.D. degree in the Power and Control Electronics Systems Group. Since 2001, he has been an Associate Professor with the Department of Communications and Signal Theory, Technical University of Catalonia, Barcelona, Spain. His current research interests are in the area of nonlinear and adaptive control for three-phase power converters.

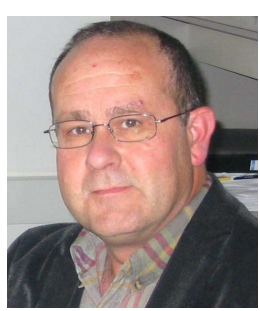

Luis Garcia de Vicuña received the Ingeniero de Telecomunicacin and Dr.Ing. degrees from the Technical University of Catalonia, Barcelona, Spain, in 1980 and 1990, respectively, and the Dr.Sci. degree from the Universit Paul Sabatier, Toulouse, France, in 1992. From 1980 to 1982 , he was an Engineer with Control Applications Company. He is currently a Full Professor in the Department of Electronic Engineering, Technical University of Catalonia, where he teaches courses on power electronics. His research interests include power electronics modeling, simulation and control, active power filtering, and high-power-factor ac/dc conversion.

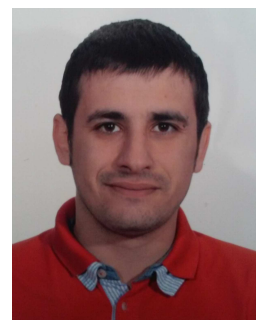

Javier Morales received the B.S. degree in electronics engineering and the M.S. degree in automation and industrial electronics from the Technical University of Catalonia, Barcelona, Spain, in 2012 and 2014, respectively. Currently, he is working towards the Ph.D. degree in electronic engineering at the Technical University of Catalonia. His research interests include automatic control and power electronics.

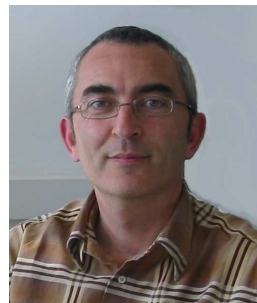

Miguel Castilla received the B.S., M.S. and Ph.D. degrees in telecommunication engineering from the Technical University of Catalonia, Barcelona, Spain, in 1988, 1995, and 1998, respectively. Since 2002, he has been an Associate Professor in the Department of Electronic Engineering, Technical University of Catalonia, where he teaches courses on analog circuits and power electronics. His research interests are in the areas of power electronics, nonlinear control, and renewable energy systems. 


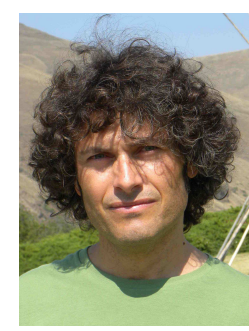

Jaume Miret (M98) received the B.S. degree in telecommunications, M.S. degree in electronics, and $\mathrm{Ph} . \mathrm{D}$. degree in electronics from the Universitat Politecnica de Catalunya, Barcelona, Spain, in 1992, 1999, and 2005, respectively. From 1993 to 2011, he was an Assistant Professor in the Department of Electronic Engineering, Universitat Politecnica de Catalunya, Spain. Since 2011 he has been an Associate Professor in the Universitat Politecnica de Catalunya, where he teaches courses on digital design and circuit theory. His research interests include dc-to-ac converters, active power filters, and digital control 